\title{
Photoacoustic viscoelasticity imaging dedicated to mechanical characterization of biological tissues
}

\author{
Yujiao Shi*, Fen Yang and Qian Wang \\ Ministry of Education Key Laboratory of Laser Life \\ Science, College of Biophotonics, South China \\ Normal University Guangzhou, 510631, P. R. China \\ Institute of Laser Life Science, College of Biophotonics \\ South China Normal University, Guangzhou \\ 510631, P. R. China \\ *shiyuj@scnu.edu.cn
}

Received 28 February 2017

Accepted 9 May 2017

Published 16 June 2017

\begin{abstract}
Since changes in mechanical properties of biological tissues are often closely related to pathology, the viscoelastic properties are important physical parameters for medical diagnosis. A photoacoustic (PA) phase-resolved method for noninvasively characterizing the biological tissue viscoelasticity has been proposed by Gao et al. [G. Gao, S. Yang, D. Xing, "Viscoelasticity imaging of biological tissues with phase-resolved photoacoustic measurement," Opt. Lett. 36, 3341-3343 (2011)]. The mathematical relationship between the PA phase delay and the viscosity-elasticity ratio has been theoretically deduced. Moreover, systems of PA viscoelasticity (PAVE) imaging including PAVE microscopy and PAVE endoscopy were developed, and high-PA-phase contrast images reflecting the tissue viscoelasticity information have been successfully achieved. The PAVE method has been developed in tumor detection, atherosclerosis characterization and related vascular endoscopy. We reviewed the development of the PAVE technique and its applications in biomedical fields. It is believed that PAVE imaging is of great potential in both biomedical applications and clinical studies.
\end{abstract}

Keywords: Photoacoustic imaging; viscoelasticity; phase detection; atherosclerosis.

\section{Introduction}

As one of the fastest growing imaging technologies, photoacoustic (PA) imaging provides excellent opportunities for in vivo characterization of disease pathophysiology. ${ }^{1-19}$ It overcomes the resolution disadvantages of pure optical imaging and the contrast disadvantages of pure ultrasound imaging, benefiting from the capacity of high-resolution

\footnotetext{
*Corresponding author.
}

This is an Open Access article published by World Scientific Publishing Company. It is distributed under the terms of the Creative Commons Attribution 4.0 (CC-BY) License. Further distribution of this work is permitted, provided the original work is properly cited. 
sensing optical contrast at depths beyond the optical transport mean-free-paths. ${ }^{20-33} \mathrm{PA}$ imaging is based on the PA effect. ${ }^{34}$ When irradiated by pulsed laser, tissue absorbs the optical energy and converts it into ultrasound due to thermal expansion, which then can be detected by transducers. ${ }^{35-38}$ Conventional PA imaging is obtained by measuring the intensity of the temporal PA signals which is generally in frequency of megahertz $(\mathrm{MHz})$, where the PA amplitude reflects the optical absorption contrast and provides structural information of the absorbers. ${ }^{39}$ Owing to the fact that biological tissues are usually viscoelastic rather than elastic, there exists a phase delay between the launched PA waves and the excitation laser. In view that changes in biological tissues are often closely related to pathology, the viscoelastic properties are important physical parameters for medical diagnosis. ${ }^{40,41}$ Therefore, the PA technique that characterizes the viscoelasticity of tissues is of great potential in medical applications and clinical research. In 2011, Gao et al. proposed a method for noninvasively characterizing the viscoelasticity of biological tissue with PA phase-resolved measurements. ${ }^{42}$ The relationship between the PA phase delay and the viscosity-elasticity ratio has been theoretically deduced. Furthermore, systems of PA viscoelasticity (PAVE) imaging including PAVE microscopy and PAVE endoscopy (PAVEE) were developed. Meanwhile, high-contrast PA phase-resolved viscoelastic images have been successfully achieved. The PAVE imaging technique has been applied to tumor detection, ${ }^{43}$ atherosclerosis characterization ${ }^{44-46}$ and vascular endoscopy. ${ }^{47}$ It is believed that PAVE imaging has great potential in both biomedical applications and clinical studies. Here, we review the development of the PAVE technique and its applications in biomedical fields.

\section{Method of the PAVE Imaging}

The principle of PAVE method is presented in Fig. 1(a). When tissues are irradiated by sinusoidal modulated laser with a laser intensity of $I_{0}$ and a modulation frequency of $\omega$, optical absorption by the tissues induces a sine-modulated temperature variation with a form of $T=T_{0} \exp (i \omega t)$. This sinemodulated temperature variation leads to sinusoidal periodic thermal expansion, which then induces sinusoidal PA wave emission with frequency equal

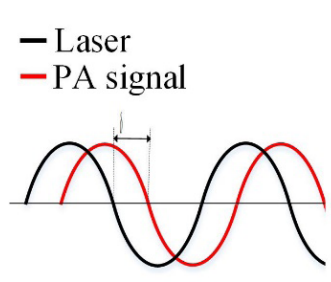

(a)

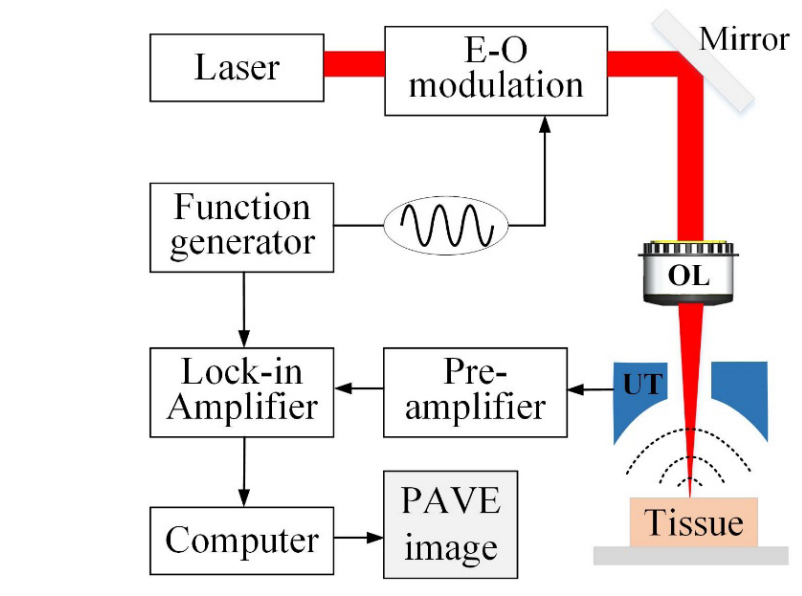

(c)

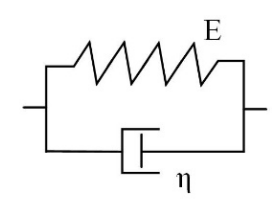

$\mathrm{KV}$ model

(b)
Fig. 1. (a) Principle of the PAVE method. (b) Sketch diagram of the KV model. (c) System of the PAVE imaging.

to the modulated frequency. Meanwhile, given the fact that the Grüneisen parameter can be considered to be a constant for biological tissues, the amplitude of the produced PA signal is proportional to the laser energy. The sinusoidal periodic heating in the region induces local thermal stress, where strain is then generated. Owing to the damping effect of the viscoelastic tissues, the PA wave alternates periodically with the modulated laser intensity with a tissue-specific phase lag, which can be modeled by the rheological Kelvin-Voigt (KV) model $^{48}$ :

$$
\varepsilon(t)=\varepsilon_{A} \exp (i(\omega t+\delta))
$$

Here, $\varepsilon$ is the strain, $\varepsilon_{A}$ is the complex strain amplitude; $\delta$ is the phase delay. As shown in Fig. 1(b), according to the KV model, the tissue-specific phase lag between the incident laser and the produced PA signal can be expressed as $^{49}$

$$
\delta=\arctan \left(\frac{\eta}{E} \omega\right)
$$

Here, $E$ represents the Young's modulus and $\eta$ represents the viscosity coefficient. As can be clearly 
seen from Eq. (2), the phase delay $\delta$ is closely related to the viscoelastic properties of biological tissues. By detecting this phase delay, we can obtain the tissues' viscoelasticity for imaging and diagnosis.

Figure 1(c) shows the experimental setup of the PAVE imaging. A CW laser ( $808 \mathrm{~nm}$ wavelength) was used as the excitation source. ${ }^{42}$ The $\mathrm{CW}$ laser was intensity-modulated by an electrooptic modulator at a frequency of $50 \mathrm{kHz}$ with a $90 \%$ modulation depth. A sinusoidal signal from a function generator was applied to the electrooptic modulator, where the sinusoidal signal was simultaneously used as the reference signal to the lock-in amplifier. The laser is focused with a focus lens and illuminates the tissues. The produced PA signal was detected by a homemade hollow focusing ultrasound transducer with a bandwidth of $20 \mathrm{kHz}$ and $50 \mathrm{kHz}$ central frequency. The outer diameter and the hole size of the hollow transducer are $4 \mathrm{~cm}$ and $1 \mathrm{~cm}$, respectively. The PA signals received by the transducer are firstly preamplified and then transmitted to the lock-in amplifier. In experiments, the samples were placed in a custom-made container and acoustically coupled with distilled water. In the PAVE imaging process, the phase delay was then collected by a computer. By mechanically scanning the tissue, the two-dimensional viscoelasticity images then could be obtained.

To verify the capability of this method in viscoelasticity measurement, six agar phantoms with different densities were used to simulate tissues with different viscoelastic properties. As shown in Fig. 2(a), the phase delay between the PA wave and the intensity-modulated laser decreases with the increase in density of the sample. The PAVE results well agree with the results obtained by the rheometer. As the fitting lines of the two groups' data are generally parallel, it indicates that the PA phase delay can be considered to be related to the viscoelastic properties of samples, and thus the proposed theory for the PAVE method is reasonable. In order to demonstrate that the phase delay is independent of the optical absorption of the samples, phantoms made by agar with different optical absorptions are performed by the PAVE system. The optical absorptions of these agar phantoms are controlled by the interfusing of ink. As shown in Fig. 2(b), the PA phase delays keep constant for agar with certain density, which indicates that the PAVE measurement is independent of the optical absorption coefficients.

The imaging capability of this method was verified by tissue-mimicking phantoms with different absorption coefficients and viscoelastic coefficients, as shown in Fig. 3. ${ }^{43}$ Figure 3(a) shows the photography of four tissue-mimicking agar phantoms. The samples marked as "a," "b," "c" and "d" contain gelatin with the concentrations of $4 \%, 4 \%$, $7 \%$ and $7 \%$, respectively. The samples marked as "b" and "d" were interfused of ink with proportions of $4 \%$ and $8 \%$. The optical absorption coefficients at $808 \mathrm{~nm}$ were tested to be $1.15 \mathrm{~cm}^{-1}$ and $1.79 \mathrm{~cm}^{-1}$, respectively, by the UV-vis spectrometer (Lambda 35, PE, USA). Figure 3(b) shows the PA images of these samples, which are very sensitive to the variations of their optical absorption coefficients. The samples marked as "b" and "d" presented bright images, because their optical absorptions are higher

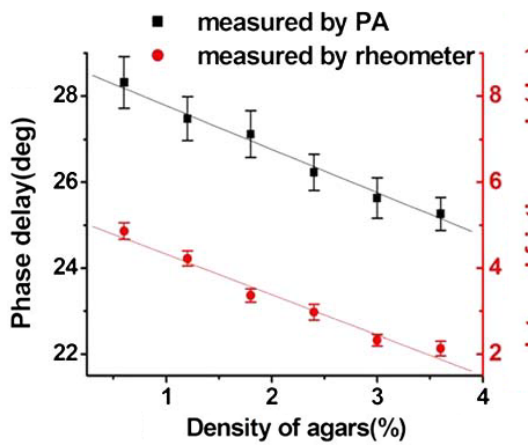

(a)

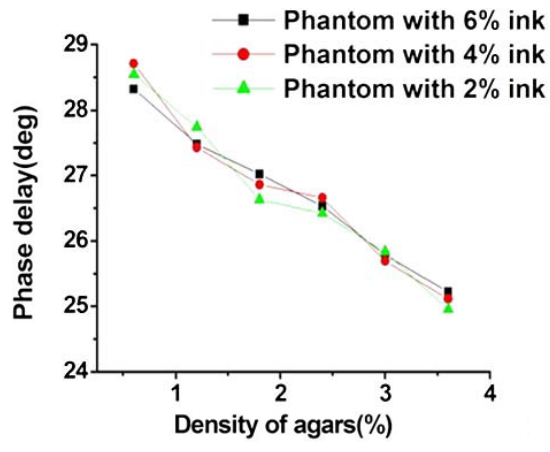

(b)

Source: Reproduced with permission from Ref. 42.

Fig. 2. (a) Comparison between the phase delay of the PA wave and the internal friction angle measured by rheometer. The values of phase delays were averaged over 16 tests. (b) Phase delays obtained by PA measurement from agars with different absorption coefficients. 
(a)

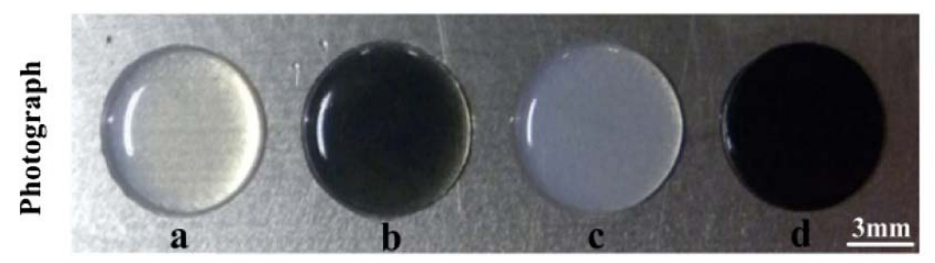

(b)

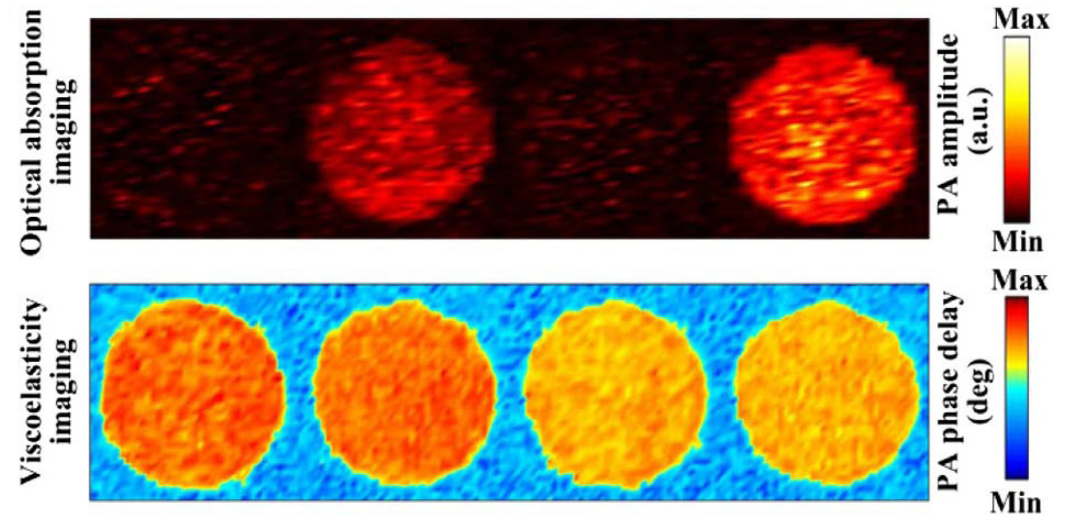

(d)

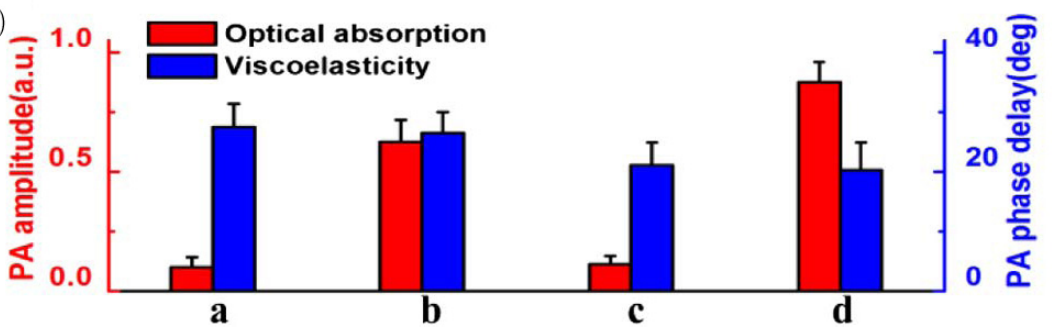

Source: Reproduced with permission from Ref. 43.

Fig. 3. (a) Photograph of the imaging samples. (b) Optical absorption image and (c) viscoelasticity image of the tissue-mimicking phantoms. (d) The averaged amplitude and phase delay of the PA signal from each phantom.

than the samples marked as "a" and "c". The PA amplitudes of samples "a" and "c" were about $18.3 \mu \mathrm{V}$ and $20.4 \mu \mathrm{V}$, respectively, where the averaged amplitude of the noise signal was about $10.4 \mu \mathrm{V}$. Therefore, the signal-to-noise ratio (SNR) of samples "a" and "c" was about $2: 1$, which was large enough for the PA lock-in measurement. The PAVE images of the four samples are shown in Fig. 3(c), which can be clearly identified for samples with different gelatin concentrations, because the viscoelastic coefficients of the samples were different. Figure 3(d) showed the mean value and standard deviation of the PA amplitude and phase delay of the four samples. Results indicated that the PA imaging and the PAVE method could be easily combined in one system and had the ability to compensate each other.

In view of the importance of the mechanical properties of tissues for disease detection, we performed a tissue imaging to verify the biological tissue imaging ability of the method. Three kinds of tissues (liver, muscle and fat from a pig) have been imaged by the PAVE method, and the experimental result is presented in Fig. 4. It is demonstrated in Fig. 4(a) that fat and muscle show lower viscosity-elasticity ratios compared with that of liver, which also well agrees

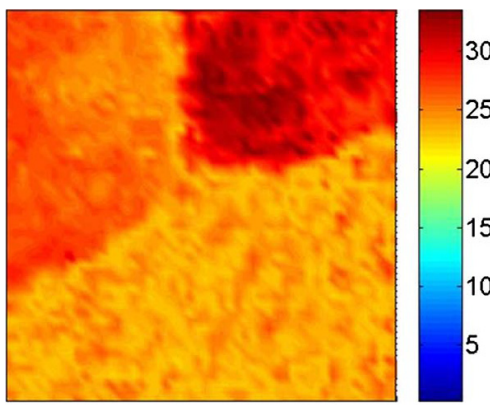

(a)

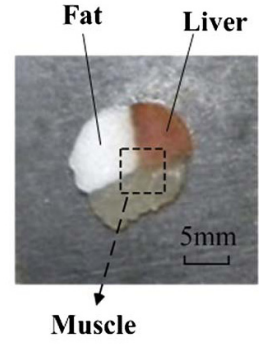

(b)
Source: Reproduced with permission from Ref. 42.

Fig. 4. (a) PAVE imaging of sample. (b) Photograph of sample; the dashed frame is the PA scanning area. 
with the rheometer results. The composite sample can be clearly revealed by PAVE imaging, and this method provides enough contrast for distinguishing different tissues. Figure 4(b) shows the tissue samples and the scanning area is marked in this figure. These PAVE results of biological tissues demonstrated that the PAVE technique has potential for biological applications and disease detection.

\section{Applications of PAVE Imaging}

PAVE imaging has wide range of biological applications, especially it can be used as an alternative method for characterizing diseases whose mechanical properties possess obvious changes. Moreover, in view that PAVE imaging is a derivative method to PA technique, it has the in vivo imaging capability and can be easily integrated into the existing techniques such as PA microscopy and PA endoscopy. The PAVE imaging technique has been applied to tumor detection, ${ }^{43}$ atherosclerosis characterization $^{44-46}$ and vascular endoscopy. ${ }^{47}$

\subsection{PA VE method for in vivo tumor detection}

As one of the most deadly diseases, tumors may be accompanied by mechanical change during their

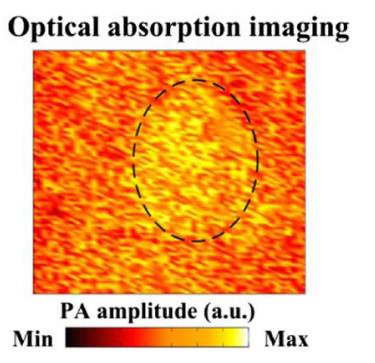

(a)

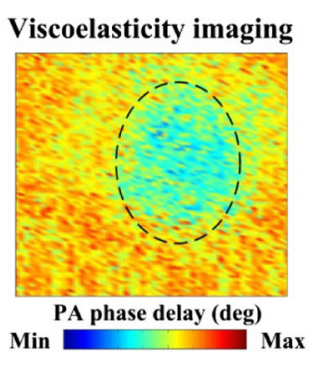

(b)

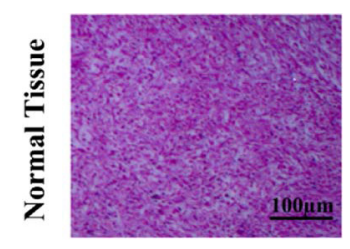

(e) development. ${ }^{50-52}$ Thus, PAVE imaging is much suited to detection of these diseases.

In vivo experiments with murine EMT6 tumor have been performed to demonstrate the feasibility of the PAVE imaging for tumor detection. ${ }^{43}$ The mouse was secured by a clamp on the $x-y$ twodimensional scanning stage. The mouse back was smeared with ultrasound coupling fluid, and was attached to the glass slide on the bottom of the water tank. The PA image and the PAVE image of the murine EMT6 tumor are shown in Fig. 5. The tumor profile could be visualized in the PA image in Fig. 5(a), and the PA signal amplitude of the tumor tissue is slightly higher than that of its surrounding tissue. While in the PAVE image shown in Fig. 5(b), the tumor region shows sufficiently high contrast to the surrounding normal tissue, owing to the fact that tumors are usually stiffer than normal tissues. ${ }^{53}$ The combination of PA imaging and PAVE imaging can provide comprehensive details for pathological diagnosis of diseases. Figure 5(c) gives the photograph of the mouse used in the experiments, where the imaging results correspond well with the mouse photograph. The dashed frame was the imaging area of Figs. 5(a) and 5(b). Figure 5(d) shows the statistical results of the mean value and standard deviation of the phase delay and the PA signal

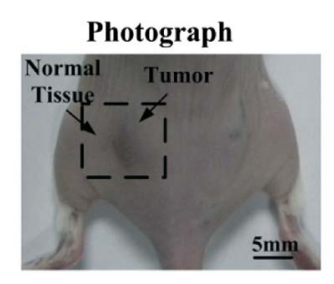

(c)

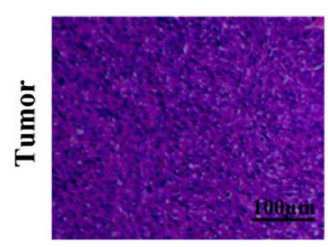

(f)

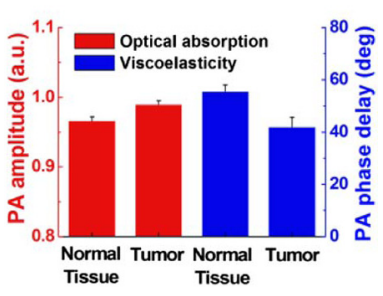

(d)

Source: Reproduced with permission from ref. 43.

Fig. 5. (a) Optical absorption image and (b) viscoelasticity image of the tumor. (c) Photograph of the tumor and the dashed frame is the scanning area. (d) The averaged amplitude and phase delay of the PA signal from the marked tumor region compared to the normal tissue. (e), (f) Hematoxylin-eosin stains of the normal tissue and tumor tissue. 
amplitudes within and outside of the dashed oval circle, which correspond to the tumor area and normal tissues, respectively. The result shows that, in the PA and PAVE imaging, tumor tissues can be easily recognized from normal tissue. Histological sections of tumor and normal tissue were sliced, which then were stained with hematoxylin-eosin (HE). The histological results are shown in Figs. 5(e) and $5(\mathrm{f})$. Compared with normal tissue, the tumor tissue appears with densely stained nuclei. These results demonstrated that the PAVE method has the ability of providing biomechanical information of tumor tissues, which thus can be used as a useful technique for medical diagnosis.

\subsection{PA VE method for atherosclerosis characterization}

Observations from animal and human models of atherosclerosis suggest that the plaque development is initiated by lipid accumulation in the arterial wall extracellular matrix, which leads to activation of inflammation and intimal fibrosis. ${ }^{54,55}$ Atherosclerotic plaques with large lipid necrotic cores and thin fibrous caps are found to be more susceptible to rupture, which is responsible for the development of the majority of acute cardiovascular events. ${ }^{56}$ The plaque's stability is usually related to its morphology and histological composition. Therefore, accurate identification of plaque may allow the detection of vulnerable plaques before they rupture. As the risk of plaque rupture is directly linked to the

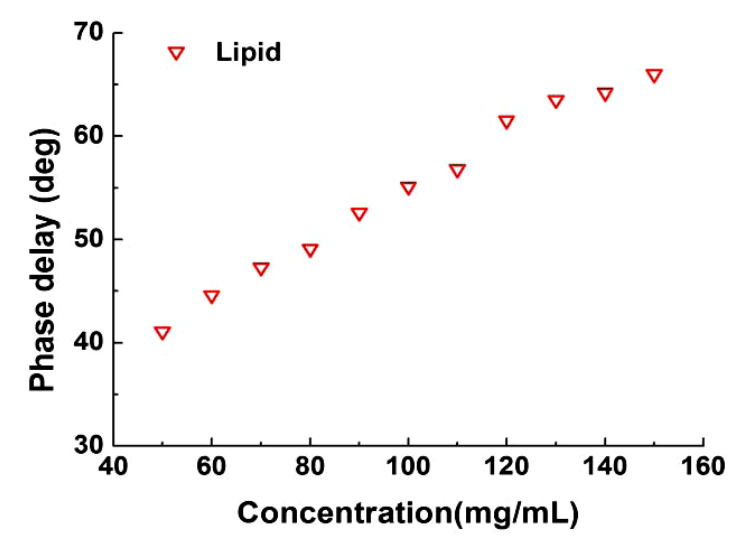

(a) mechanical properties of the plaques, ${ }^{57}$ a comprehensive understanding of the mechanical properties of the plaques can advance understanding of the pathology of atherosclerotic diseases. ${ }^{58}$ The PAVE imaging technique has been applied for precisely evaluating the viscoelastic properties of atherosclerotic plaques, which could provide new insights into diagnosis of cardiovascular-related diseases. ${ }^{44-47}$

\subsubsection{Plaque-mimicking phantom test}

In order to verify the ability of PAVE imaging for identification of plaque lipids, plaque-mimicking phantoms were studied experimentally, as shown in Fig. 6. Lipid, which is the main material in early plaque lesions, was mixed with gelatin in different concentrations to simulate atherosclerotic plaques of different stages during the progress of plaques. Figure 6(a) shows the relation between the lipid content and phase delay. PAVE images of four phantoms with different lipid concentrations are clearly shown in Fig. 6(b). The observed phase delay of the PA signals increased significantly with the increasing density of the lipid. The results indicate that PAVE imaging has great potential for atherosclerotic diseases characterization.

\subsubsection{Rabbit plaque characterization by PAVE method}

We demonstrated the PAVE imaging ability in rabbit plaques. The result of the viscoelasticityinduced phase delay of a line-scanning along a

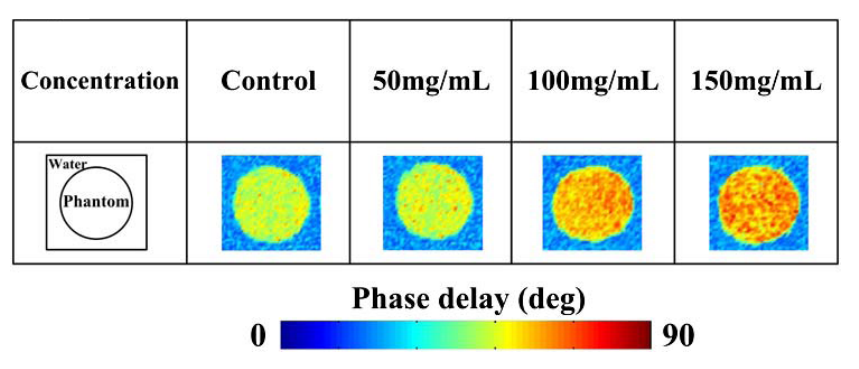

(b)

Source: Reproduced with permission from Ref. 46.

Fig. 6. (a) Relation between the lipid concentration and phase delay. (b) PAVE imaging of phantoms containing various concentrations of lipid. 


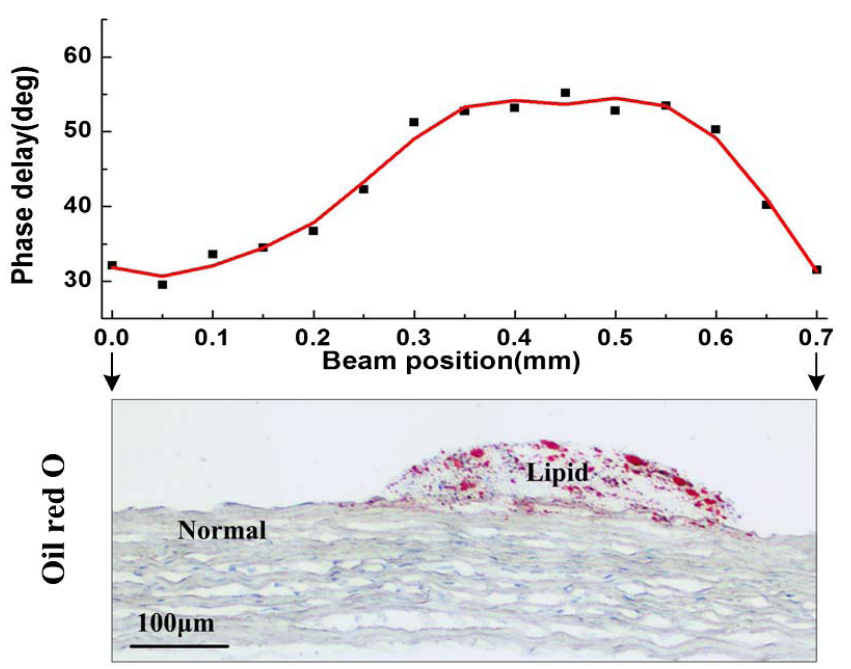

Source: Reproduced with permission from Ref. 46.

Fig. 7. Variation in phase delay as the laser beam scans across the vessel wall from the normal region to the lipid lesion.

plaque is shown in Fig. 7. As the laser beam scanned across the plaque, the phase delay varied significantly with tissue type, where the phase delay was low in the normal regions and high in the lipid-rich regions. Thus, the atherosclerotic plaque can be clearly differentiated from the normal arterial wall because of the high viscoelasticity of lipid. The related Oil Red O stain results of the sections clearly showed the surface composition changes of the

(a)
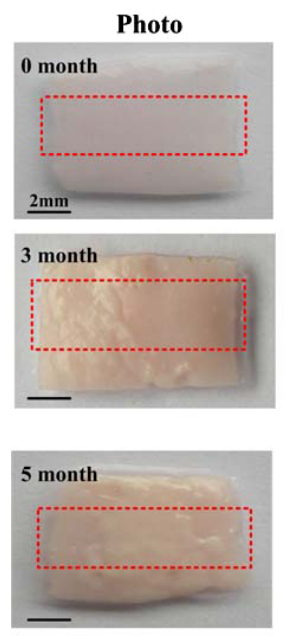

(b)
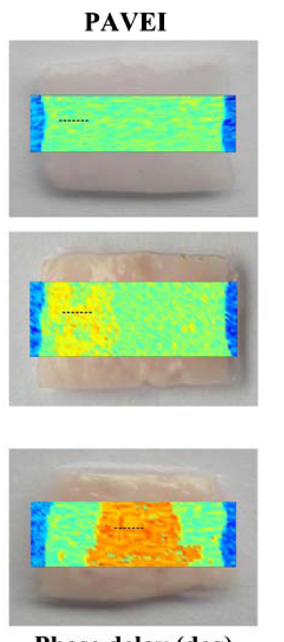

Phase delay (deg) $0 \square 90$

artery. The correspondence between the histological sections and the PAVE result demonstrated that the lipid can provide enough contrast with normal tissues in the PAVE technique.

\subsubsection{PAVE imaging for plaque staging}

A representative example was used to demonstrate the ability of the PAVE imaging system for characterizing atherosclerotic plaques. Figure 8 shows that the PAVE technique has the potential to characterize the plaque composition, which is of crucial importance for monitoring the development progress of atherosclerotic plaques. Figure 8(a) shows the region of the arterial lumen in a healthy rabbit and the atherosclerotic lesions found in highfat/high-cholesterol-feeding three- and five-monthold rabbits, respectively. The arterial plaques showed abundant lipid-rich structures differing in appearance from the healthy vessels. Results in Fig. 8(b) presented that the average phase delay obtained from images of the lumen surface in the healthy region is significantly lower than that in the atherosclerotic regions. Meanwhile, the average phase delay from the plaque found in the threemonth-old rabbit is much lower than that found in the five-month-old rabbit. Therefore, the phase delay from regions of plaque increased with the age of the rabbits, which indicated the overall burden of

(c)
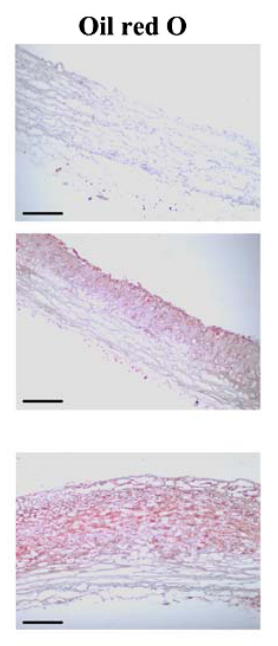

(d)

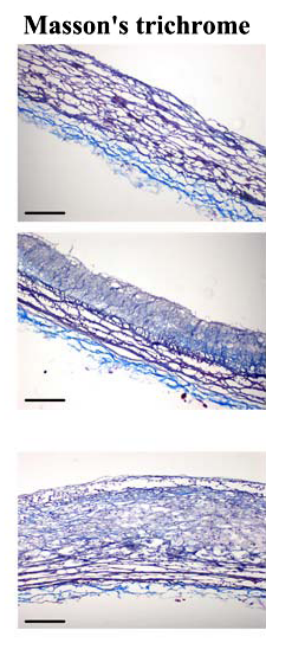

Source: Reproduced with permission from Ref. 46.

Fig. 8. (a) Photos and (b) representative PAVE images acquired from the luminal surface of the rabbit arteries, with healthy lumen (top row) early atherosclerotic plaque (middle row) and atherosclerotic plaque with higher lipid content (bottom row); (c) the Oil Red O and (d) Masson's trichrome stain images of the areas in the corresponding dashed rectangles. Bars $=300 \mu \mathrm{m}$. 
plaque in the aorta. The lipid accumulation is clearly shown by the histological sections in Fig. 8(c). The lipid content increased from a basal average of $0.018 \pm 0.002 \mathrm{IOD} /$ area in a healthy artery to $0.108 \pm 0.005 \mathrm{IOD} /$ area in the fatty artery after three months of a high-fat/high-cholesterol diet. For five-month-old rabbits, the $0.147 \pm 0.008$ IOD/area value of the lipid plaque showed a significant difference compared with other groups. The increase of the average viscosity-elasticity ratio was predominantly due to the accumulated lipid and the decrease of collagen content [Fig. 8(d)], which plays an important role in the development of vulnerable plaque.

\subsection{Combined $P A$ and $P A V E$ imaging for precise and multi-information plaque characterization}

As PAVE imaging is a derivative method to PA technique, it can be easily integrated into the existing techniques such as PA microscopy. The combined PA-PAVE technique could provide structural, componential and biomechanical information of plaques, and thus has received much attention in biomedical fields. To demonstrate the feasibility of combined PA-PAVE technique for biomedical application, an atherosclerotic tissue with a fatty streak harvested from a 15-week-old high-fat/high-cholesterol diet feeding rabbit was experimentally tested. ${ }^{45}$ Laser was illuminated on the surface of the atherosclerotic sample. A highfrequency ultrasound transducer with central frequency of $75 \mathrm{MHz}$ was used in the system to image

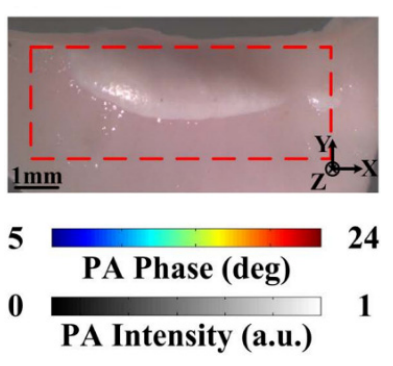

(a)

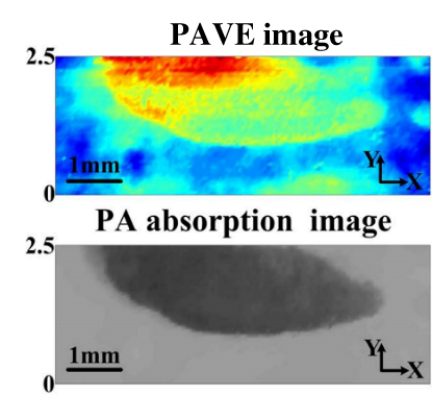

(b) the thin-layer structure of vascular wall. The photograph of the atherosclerotic tissue was shown in Fig. 9(a), and tissue within the red dashed frame was the scanning region in ex vivo examination. The en-face PAVE and PA images shown in Fig. 9(b) both distinguished the morphology of the fatty streak and corresponded well with the sample. The fatty tissue (main component is lipid) suggested higher viscoelasticity and slightly lower optical absorption than those of the surrounding normal tissue (main component is collagen), which is consistent with the fact that the viscoelasticity of lipid is greater than that of collagen ${ }^{59}$ and the optical absorption of lipid is lower than that of collagen at $1064 \mathrm{~nm} .{ }^{60}$ The inhomogeneity of viscoelasticity and absorption distribution at the atherosclerotic tissue came from the different degrees of lipid accumulation. After PA experiment, the specimen was sliced and evaluated with cross-sectional Oil Red O staining.

The integrated PA-PAVE image along the sectional direction and the corresponding histological sections were shown in Fig. 9(c). In the fatty streak, the intima thickening resulting from lipid accumulation exhibited high viscoelasticity and dense Oil Red O staining, where the most severe intima-media thickening was about $0.7 \mathrm{~mm}$ and was highly consistent with the histology. The imaging depth of the PA-PAVE system was about $1.5 \mathrm{~mm}$. These integrated PA-PAVE sections allowed complementary visualization of en-face viscoelasticity distribution and in-depth structural anatomy of the fatty streak, where the distortion of viscoelasticity distribution of the surface may be an early warning of plaque rupture, and the degree of intima

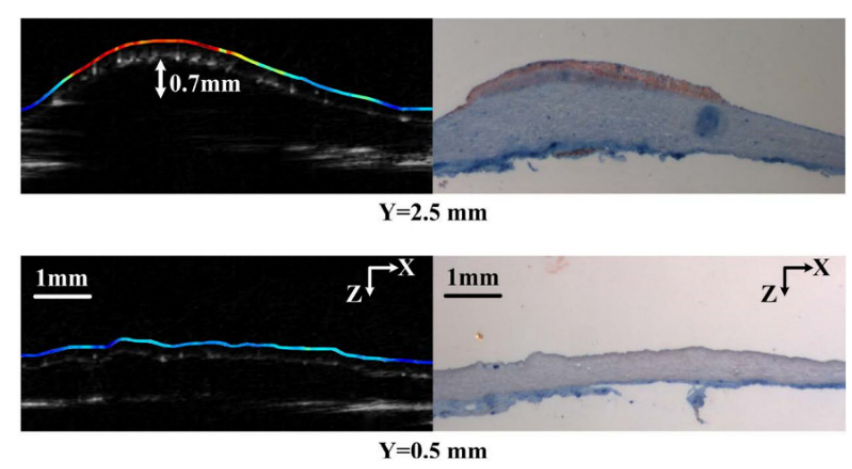

(c)

Source: Reproduced with permission from ref. 45.

Fig. 9. (a) Photograph of the atherosclerotic tissue with a fatty streak, the region within dashed frame is the scanning area. (b) Enface PAVE and absorption images. (c) Integrated PA sections and corresponding histologies. The sections were stained with Oil Red $\mathrm{O}$ (red) to evaluate lipid accumulation and counterstained with hematoxylin (blue) to visualize cell nuclei. 
thickening relates to the lesion extent. These experimental results demonstrated the feasibility of PA-PAVE technique for accurate medical evaluation of atherosclerosis.

\subsection{PA VE endoscopy}

To realize the clinical application of the PAVE technique, a PAVE endoscope (PAVEE) ${ }^{45}$ is developed for potential applications of intraluminal tissues such as blood vessels, esophagus, rectum and cervical. The experimental setup of the PAVEE was shown in Fig. 10. A quasi-continuous $1064 \mathrm{~nm}$ laser with a pulse width of $22 \mathrm{~ns}$ and $65 \mathrm{kHz}$ of repetition frequency was used as the excitation source. The collimated laser was focused by a microscope objective and coupled into an optical fiber. The distal end of the optical fiber passed through a hollow motor and ultrasound transducer with $65 \mathrm{kHz}$ central frequency to irradiate the laser on a customized parabolic reflector, which could simultaneously reflect and focus the laser. The reflector was fixed on a rotating motor to realize cross-sectional scanning. The transducer and scanning mirror were held in a nickel tube, which had opened windows for laser excitation and detecting PA signals. All the components were installed on a motorized pullback stage to realize a longitudinal scanning. The distance between the laser focus and transducer was maintained strictly constant during the data acquisition. The generated PA signals were acoustically coupled with distilled water and detected by the ultrasound transducer, which then were delivered to a preamplifier, and calculated by a lock-in detector to obtain the phase delay. The data were collected and analyzed on a computer which

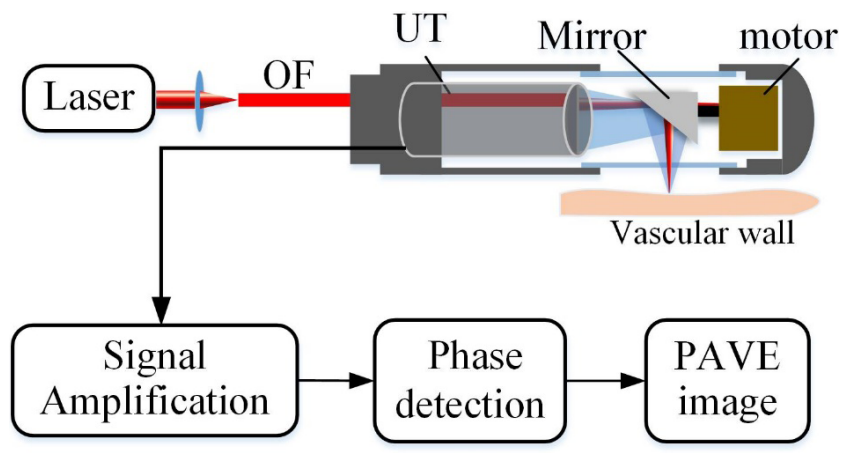

Fig. 10. Schematic of the experimental setup for PAVEE; MO: microscopic objective, UT: ultrasound transducer and OF: optical fiber. controlled the motorized scanner with a LabVIEW program simultaneously.

To further validate the feasibility of PAVEE for biomedical application, ex vivo experiments on vessels harvested from a three-month-old high-fat/ high-cholesterol diet feeding rabbit were conducted. The vessel-shaped sample with a $19 \mathrm{~mm}$ luminal diameter was prepared, then the PAVEE imaging was performed in the lumen of the sample. PAVEE image and en-face viscoelasticity distribution were shown in Fig. 11(a). The longitudinal PAVEE image enabled an overall viscoelastic evaluation of the luminal sample. The atherosclerotic tissue showed an inhomogeneity of PA viscoelasticity distribution, which was relatively uniform in the normal tissue. As expected, the lipid-rich regions presented higher viscoelasticity and phase delay. The PAVEE result verified a good correlation with both the sample morphology and the corresponding en-face PA viscoelasticity image. After experiment, the specimen was cross-sectionally sliced and stained with Oil Red. As shown in Fig. 11(b), the cross-sectional PAVEE section and histological staining at $Z=0.8 \mathrm{~mm}$ demonstrated a good correlation between PAVEE and histology, where lipid-rich plaques in atherosclerotic segment suggested high viscoelasticity and dense Oil Red staining. Our experimental results proved that the PAVEE has the potential for sensitively differentiating atherosclerosis and accurate medical evaluation in situ.

\section{Discussion and Conclusion}

In the PAVE imaging system, in order to obtain high data accuracy of the measurement, the time constant of the lock-in amplifier is set to be $300 \mathrm{~ms}$. The acquisition time required for PAVE image of Fig. 4(a) is about $45 \mathrm{~min}$. The imaging speed can be improved by reducing the time constant of the lockin amplifier and improving the speed of the scanning platform. During the measurement, the time of laser exposure in each scanning point was about $300 \mathrm{~ms}$ and the laser-induced temperature increase was approximately $0.2^{\circ} \mathrm{C}$. Thus, the impact of the temperature increment on the viscoelasticity could be ignored.

Elasticity and viscosity are the significant physical parameters to characterize mechanical properties of biological tissue. They can potentially be used to reflect pathological conditions. Several 

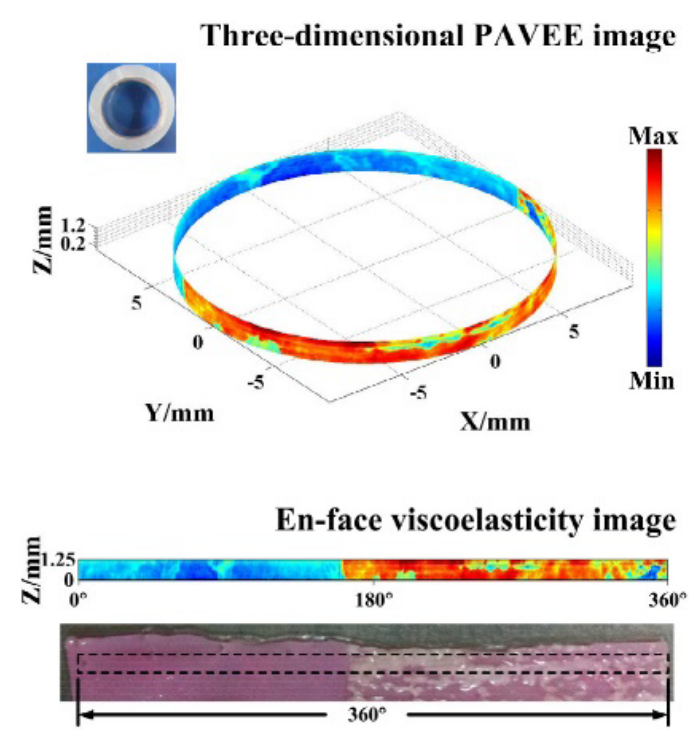

(a)

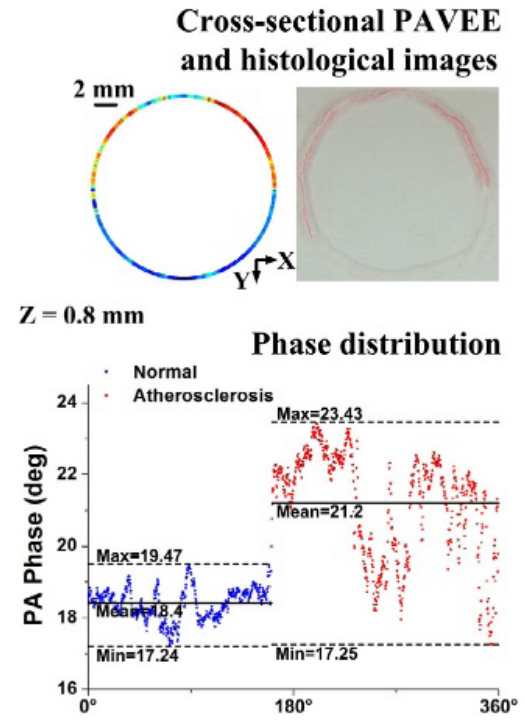

(b)

Source: Reproduced with permission from Ref. 44.

Fig. 11. (a) Longitudinal PAVEE image and en-face viscoelasticity image viewed from inside over a $360^{\circ}$ field of the sample. In the flattened tissue photograph, the region within dashed frame is the scanning area. (b) PAVEE section and corresponding histology and phase distribution at $z=0.8 \mathrm{~mm}$. The section was stained with Oil Red O (red) targeting lipid.

imaging techniques, including ultrasound elastography ${ }^{61}$ magnetic resonance elastography (MRE),${ }^{62}$ optical coherence elastography $(\mathrm{OCE})^{63}$ and PA elastography ${ }^{64}$ have been used for mapping elasticity distribution. However, we noticed that these techniques ignore the fact that biological tissues are usually considered to be viscoelastic rather than elastic. In contrast, the PAVE technique has the capacity to reveal the viscoelastic properties of tissues, which thus could provide new insights into biomechanical diagnosis with richer information.

The measured phase by our system is the phase difference between the PA signal and the reference signal used to modulate the laser, which can be considered as a sum of the viscoelasticity-related phase delay, nonradiative transition-induced phase delay and system delay. Since the relaxation time for nonradiative transition is about $10^{-11} \mathrm{~s},{ }^{65}$ it then can be neglected in our measurement. Although the system delay is difficult to measure, it can maintain stably and keep constant in the same system. However, in the PAVE imaging, as the distance between the sample and the ultrasound transducer influences the phase delay, the surface of sample needs to be smooth during experiments, which may limit its in vivo applications. It is also noticed that, as the PAVE measurement is based on phase detection by a lock-in amplifier, this method lost its capability of depth-resolved imaging, which may be complemented by conventional PA technique. Furthermore, we realized that quantitative elasticity and viscosity of the tissues cannot be resolved by the PAVE method, which hinders its biological applications. Further development of this technique is still needed to explore the possibility of breakthrough in these limitations.

In conclusion, we reviewed the development of the PAVE technique and its applications in biomedical fields, and discussed its limitations. The PAVE method has been developed in tumor detection, atherosclerosis characterization and related vascular endoscopy. Even though there are still some limitations that existed in the PAVE imaging, it is trusted that it is of great potential in both biomedical research and clinical study.

\section{Acknowledgments}

This research is supported by the National Natural Science Foundation of China (Grant Nos. 81630046, 61627827, 61331001 and 91539127), the Science and Technology Planning Project of Guangdong Province, China (Nos. 2015B020233016, 2014B020215003 and 2014A020215031), the Science and Technology Youth Talent for Special Program of Guangdong, China (Nos. 2015TQ01X882) and the Distinguished 
Young Teacher Project in Higher Education of Guangdong, China (No. YQ2015049).

\section{References}

1. M. Xu, L. V. Wang, "Photoacoustic imaging in biomedicine," Rev. Sci. Instrum. 77, 041101 (2006).

2. L. V. Wang, S. Hu, "Photoacoustic tomography: In vivo imaging from organelles to organs," Science 335, 1458-1462 (2012).

3. J. Zhang, S. Yang, X. Ji, Q. Zhou, D. Xing, "Characterization of lipid-rich aortic plaques by intravascular photoacoustic tomography," J. Am. Coll. Cardiol. 64, 385-390 (2014).

4. P. C. Li, C. R. C. Wang, D. B. Shieh, C. W. Wei, C. K. Liao, C. Poe, S. Jhan, A.-A. Ding, Y. N. Wu, "In vivo photoacoustic molecular imaging with simultaneous multiple selective targeting using antibody-conjugated gold nanorods," Opt. Express 16, 18605-18615 (2008).

5. K. Maslov, G. Stoica, L. V. Wang, "In vivo darkfield reflection-mode photoacoustic microscopy," Opt. Lett. 30, 625-627 (2005).

6. S. Yang, D. Xing, Q. Zhou, L. Xiang, Y. Lao, "Functional imaging of cerebrovascular activities in small animals using high-resolution photoacoustic tomography," Med. Phys. 34, 3294-3301 (2007).

7. A. De La Zerda, C. Zavaleta, S. Keren, S. Vaithilingam, S. Bodapati, Z. Liu, J. Levi, B. R. Smith, T.-J. Ma, O. Oralkan, Z. Cheng, X. Chen, H. Dai, B. T. Khuri-Yakub, S. S. Gambhir, "Carbon nanotubes as photoacoustic molecular imaging agents in living mice," Nat. Nanotechnol. 3, 557-562 (2008).

8. R. A. Kruger, P. Liu, Y. Fang, C. R. Appledorn, "Photoacoustic ultrasound (PAUS) - reconstruction tomography," Med. Phys. 22, 1605-1609 (1995).

9. L. Zeng, D. Xing, H. Gu, D. Yang, S. Yang, L. Xiang, "High antinoise photoacoustic tomography based on a modified filtered backprojection algorithm with combination wavelet," Med. Phys. 34, 556-563 (2007).

10. Y. Lao, D. Xing, S. Yang, L. Xiang, "Noninvasive photoacoustic imaging of the developing vasculature during early tumor growth," Phys. Med. Biol. 53, 4203-4212 (2008).

11. J. W. Kim, E. I. Galanzha, E. V. Shashkov, H. M. Moon, V. P. Zharov, "Golden carbon nanotubes as multimodal photoacoustic and photothermal highcontrast molecular agents," Nat. Nanotechnol. 4, 688-694 (2009).

12. L. Xiang, Y. Yuan, D. Xing, Z. Ou, S. Yang, F. Zhou, "Photoacoustic molecular imaging with antibody-functionalized single-walled carbon nanotubes for early diagnosis of tumor," J. Biomed. Opt. 14, 021008 (2009).

13. C. Li, L. V. Wang, "Photoacoustic tomography and sensing in biomedicine," Phys. Med. Biol. 54, R59-R97 (2009).

14. G. Huang, S. Yang, Y. Yuan, D. Xing, "Combining $\mathrm{X}$-ray and photoacoustics for in vivo tumor imaging with gold nanorods," Appl. Phys. Lett. 99, 123701 (2011).

15. Z. Chen, S. Yang, D. Xing, "In vivo detection of hemoglobin oxygen saturation and carboxyhemoglobin saturation with multiwavelength photoacoustic microscopy," Opt. Lett. 37, 3414-3416 (2012).

16. H. F. Zhang, K. Maslov, M. Sivaramakrishnan, G. Stoica, L. V. Wang, "Imaging of hemoglobin oxygen saturation variations in single vessels in vivo using photoacoustic microscopy," Appl. Phys. Lett. 90, 053901 (2007).

17. S. Yang, F. Ye, D. Xing, "Intracellular label-free gold nanorods imaging with photoacoustic microscopy," Opt. Express 20, 10370-10375 (2012).

18. B. Li, H. Qin, S. Yang, D. Xing, "In vivo fast variable focus photoacoustic microscopy using an electrically tunable lens," Opt. Express 22, 20130-20137 (2014).

19. Y. S. Chen, W. Frey, S. Kim, P. Kruizinga, K. Homan, S. Emelianov, "Silica-coated gold nanorods as photoacoustic signal nanoamplifiers," Nano Lett. 11, 348-354 (2011).

20. X. Ji, K. Xiong, S. Yang, D. Xing, "Intravascular confocal photoacoustic endoscope with dual-element ultrasonic transducer," Opt. Express 23, 9130-9136 (2015).

21. G. He, D. Xu, H. Qin, S. Yang, D. Xing, "In vivo cell characteristic extraction and identification by photoacoustic flow cytography," Biomed. Opt. Express 6, 3748-3756 (2015).

22. L. Song, K. Maslov, R. Bitton, K. K. Shung, L. V. Wang, "Fast 3-D dark-field reflection-mode photoacoustic microscopy in vivo with a $30-\mathrm{MHz}$ ultrasound linear array," J. Biomed. Opt. 13, 054028 (2008).

23. Y. Shi, H. Qin, S. Yang, D. Xing, "Thermally confined shell coating amplifies the photoacoustic conversion efficiency of nanoprobes," Nano Res. 9, 3644-3655 (2016).

24. Z. Chen, S. Yang, Y. Wang, D. Xing, "Noncontact broadband all-optical photoacoustic microscopy based on a low-coherence interferometer," Appl. Phys. Lett. 106, 043701 (2015).

25. E. Z. Zhang, J. G. Laufer, R. B. Pedley, P. C. Beard, "In vivo high-resolution 3D photoacoustic imaging of superficial vascular anatomy," Phys. Med. Biol. 54, 1035-1046 (2009). 
26. Y. Wang, D. Xu, S. Yang, D. Xing, "Toward in vivo biopsy of melanoma based on photoacoustic and ultrasound dual imaging with an integrated detector," Biomed. Opt. Express 7, 279-286 (2016).

27. Y. Liu, X. Yang, D. Zhu, R. Shi, Q. Luo, "Optical clearing agents improve photoacoustic imaging in the optical diffusive regime," Opt. Lett. 38, 4236-4239 (2013).

28. D. Xu, S. Yang, Y. Wang, Y. Gu, D. Xing, "Noninvasive and high-resolving photoacoustic dermoscopy of human skin," Biomed. Opt. Express 7, 2095-2102 (2016).

29. Z. Chen, S. Yang, D. Xing, "Optically integrated trimodality imaging system: Combined all-optical photoacoustic microscopy, optical coherence tomography, and fluorescence imaging," Opt. Lett. 41, 1636-1639 (2016).

30. S. Mallidi, T. Larson, J. Tam, P. P. Joshi, A. Karpiouk, K. Sokolov, S. Emelianov, "Multiwavelength photoacoustic imaging and plasmon resonance coupling of gold nanoparticles for selective detection of cancer," Nano Lett. 9, 2825-2831 (2009).

31. J. Zhong, S. Yang, L. Wen, D. Xing, "Imagingguided photoacoustic drug release and synergistic chemo-photoacoustic therapy with paclitaxelcontaining nanoparticles," J. Control. Release 226, 77-87 (2016).

32. H. Qin, T. Zhou, S. Yang, D. Xing, "Fluorescence quenching nanoprobes dedicated to in vivo photoacoustic imaging and high-efficient tumor therapy in deep-seated tissue," Small 11, 2675-2686 (2015).

33. J. Shah, S. Park, S. Aglyamov, T. Larson, L. Ma, K. Sokolov, K. Johnston, T. Milner, S. Y. Emelianov, "Photoacoustic imaging and temperature measurement for photothermal cancer therapy," J. Biomed. Opt. 13, 034024 (2008).

34. A. G. Bell, "On the production and reproduction of sound by light," Am. J. Sci. 118, 305-324 (1880).

35. G. J. Diebold, T. Sun, M. I. Khan, "Photoacoustic monopole radiation in one, two, and three dimensions," Phys. Rev. Lett. 67, 3384-3387 (1991).

36. A. Rosencwaig, A. Gersho, "Theory of the photoacoustic effect with solids," J. Appl. Phys. 47, 64-69 (1976).

37. C. Lou, S. Yang, Z. Ji, Q. Chen, D. Xing, "Ultrashort microwave-induced thermoacoustic imaging: A breakthrough in excitation efficiency and spatial resolution," Phys. Rev. Lett. 109, 218101 (2012).

38. Z. Ji, W. Ding, F. Ye, C. Lou, D. Xing, "Shapeadapting thermoacoustic imaging system based on flexible multi-element transducer," Appl. Phys. Lett. 107, 094104 (2015).

39. J. F. Greenleaf, M. Fatemi, M. Insana, "Selected methods for imaging elastic properties of biological tissues," Annu. Rev. Biomed. Eng. 5, 57-78 (2003).
40. T. Xydeas, K. Siegmann, R. Sinkus, U. KrainickStrobel, S. Miller, C. D. Claussen, "Magnetic resonance elastography of the breast: Correlation of signal intensity data with viscoelastic properties," Invest. Radiol. 40, 412-420 (2005).

41. J. Rogowska, N. A. Patel, J. G. Fujimoto, M. E. Brezinski, "Optical coherence tomographic elastography technique for measuring deformation and strain of atherosclerotic tissues," Heart 90, 556-562 (2004).

42. G. Gao, S. Yang, D. Xing, "Viscoelasticity imaging of biological tissues with phase-resolved photoacoustic measurement," Opt. Lett. 36, 3341-3343 (2011).

43. Y. Zhao, S. Yang, C. Chen, D. Xing, "Simultaneous optical absorption and viscoelasticity imaging based on photoacoustic lock-in measurement," Opt. Lett. 39, 2565-2568 (2014).

44. C. Chen, Y. Zhao, S. Yang, D. Xing, "Mechanical characterization of intraluminal tissue with phaseresolved photoacoustic viscoelasticity endoscopy," Biomed. Opt. Express 6, 4975-4980 (2015).

45. C. Chen, Y. Zhao, S. Yang, D. Xing, "Integrated mechanical and structural features for photoacoustic characterization of atherosclerosis using a quasi-continuous laser," Opt. Express 23, 17309-17315 (2015).

46. Y. Zhao, C. Chen, S. Yang, D. Xing, "Mechanical evaluation of lipid accumulation in atherosclerotic tissues by photoacoustic viscoelasticity imaging," Opt. Lett. 41, 4522-4525 (2016).

47. Y. Zhao, C. Chen, H. Liu, S. Yang, D. Xing, "Timeresolved photoacoustic measurement for evaluation of viscoelastic properties of biological tissues," Appl. Phys. Lett. 109, 203702 (2016).

48. L. B. Eldred, W. P. Baker, A. N. Palazotto, "Kelvin-Voigt versus fractional derivative model as constitutive relations for viscoelastic materials," AIAA J. 33, 547-550 (1995).

49. J. Chen, R. Lin, H. Wang, J. Meng, H. Zheng, L. Song, "Blind-deconvolution optical-resolution photoacoustic microscopy in vivo," Opt. Express $\mathbf{2 1}$, 7316-7327 (2013).

50. V. Swaminathan, K. Mythreye, E. T. O'Brien, A. Berchuck, G. C. Blobe, R. Superfine, "Mechanical stiffness grades metastatic potential in patient tumor cells and in cancer cell lines," Cancer Res. 71, 5075-5080 (2011).

51. R. P. Araujo, D. L. S. McElwain, "A linear-elastic model of anisotropic tumour growth," Eur. J. Appl. Math. 15, 365-384 (2004).

52. A. Lyshchik, T. Higashi, R. Asato, S. Tanaka, J. Ito, J. J. Mai, C. Pellot-Barakat, M. F. Insana, A. B. Brill, T. Saga, M. Hiraoka, K. Togashi, "Thyroid gland tumor diagnosis at US elastography," Radiology 237, 202-211 (2005). 
53. M. J. Paszek, N. Zahir, K. R. Johnson, J. N. Lakins, G. I. Rozenberg, A. Gefen, C. A. Reinhart-King, S. S. Margulies, M. Dembo, D. Boettiger, D. A. Hammer, V. M. Weaver, "Tensional homeostasis and the malignant phenotype," Cancer Cell 8, 241-254 (2005).

54. A. J. Lusis, "Atherosclerosis," Nature 407, 233-241 (2000).

55. C. K. Glass, J. L. Witztum, "Atherosclerosis: The road ahead," Cell 104, 503-516 (2001).

56. R. Virmani, A. P. Burke, A. Farb, F. D. Kolodgie, "Pathology of the vulnerable plaque," J. Am. Coll. Cardiol. 47, C13-C18 (2006).

57. C. L. De Korte, S. G. Carlier, F. Mastik, M. M. Doyley, A. F. W. Van Der Steen, P. W. Serruys, N. Bom, "Morphological and mechanical information of coronary arteries obtained with intravascular elastography: Feasibility study in vivo," Eur. Heart J. 23, 405-413 (2002).

58. Q. Lu, K. Ganesan, D. T. Simionescu, N. R. Vyavahare, "Novel porous aortic elastin and collagen scaffolds for tissue engineering," Biomaterials 25, 5227-5237 (2004).

59. L. W. Lake, C. D. Armeniades, "Structure-property relations of aortic tissue," ASAIO J. 18, 202-208 (1972).
60. L. Yoo, V. Gupta, C. Lee, P. Kavehpore, J. L. Demer, "Viscoelastic properties of bovine orbital connective tissue and fat: Constitutive models," Biomech. Model. Mechanobiol. 10, 901-914 (2011).

61. J. Ophir, I. Cespedes, H. Ponnenkanti, Y. Yazdi, X. Li, "Elastography: A quantitative method for imaging the elasticity of biological tissues," Ultrason. Imaging 13, 111-134 (1991).

62. R. Muthupillai, D. J. Lomas, P. J. Rossman, J. F. Greenleaf, R. L. Ehman, "Magnetic resonance elastography by direct visualization of propagating acoustic strain waves," Science 269, 1854-1857 (1995).

63. R. K. Wang, Z. H. Ma, S. J. Kirkpatrick, "Tissue Doppler optical coherence elastography for real time strain rate and strain mapping of soft tissue," Appl. Phys. Lett. 89, 144103 (2006).

64. P. F. Hai, J. J. Yao, G. Li, C. Y. Li, L. V. Wang, "Photoacoustic elastography," Opt. Lett. 41, 725-728 (2016).

65. M. Takezaki, N. Hirota, M. Terazima, "Nonradiative relaxation processes and electronically excited states of nitrobenzene studied by picosecond time-resolved transient grating method," J. Phys. Chem. A 101, 3443-3448 (1997). 